\title{
Die Cyperussäule.
}

\section{Von LUdwig Borchard'.}

Seit dem Erscheinen der "ägyptischen Pflanzensäule " im Jahre 1897 habe ich Gelegenheit gehabt, eine weitere Säulenart, deren Vorbild gleichfalls unter den ägyptischen Wasserptlanzen zu suchen ist, zu identifizieren. Um den Raum dieser Zeitschrift nicht zu sehr in Anspruch zu nehmen, mag es hier genügen, kurz das Pflanzenvorbild zu beschreiben, einige Beispiele der Pflanze in der Ornamentik zu geben und endlich die Säulenart in wenigen Beispielen vorzuführen. Die hier (Abb. 1 u. 2) nach einigen Aufnahmen aus dem Fajum wiedergegebene Pflanze ist nach Schwernfurti Cyperus alopecuroïdes (Rottb.).

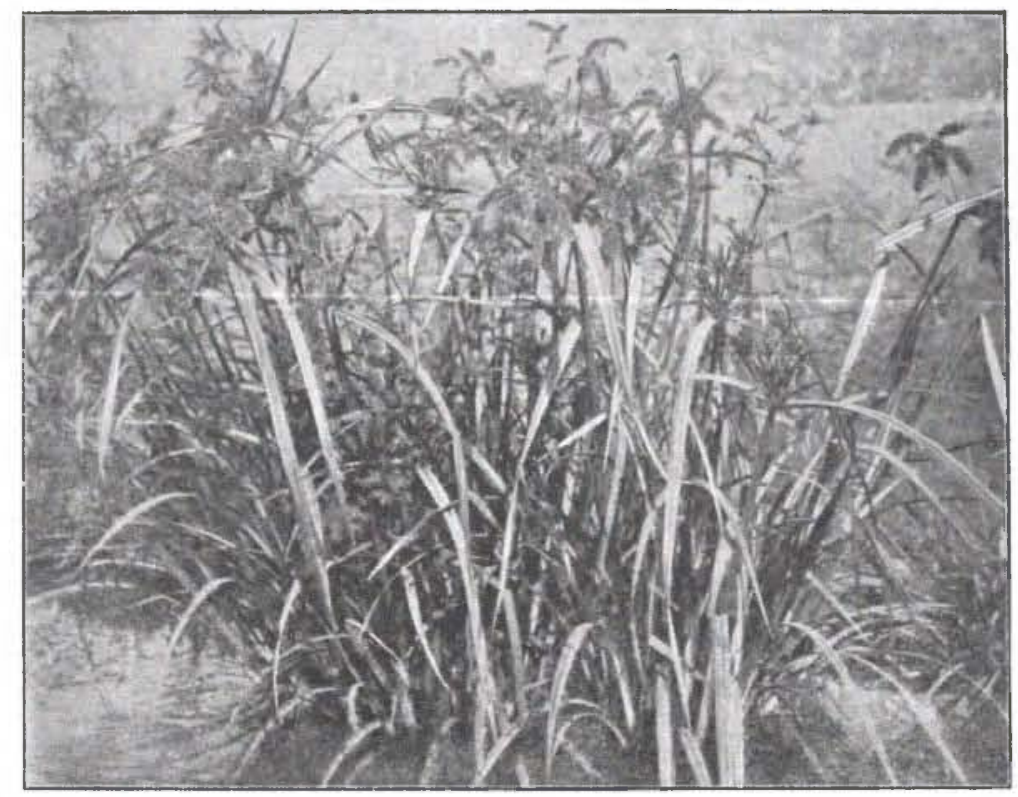

Abb. 1: Busch von Cyperus alopecuroüdes.

Für die Aufnahme der Einzelpflanze (Abb. 2) konnte ich leider keine volleren Exemplare auftreiben. Man sieht aber auch an den hier gegebenen das Charakteristische deutlich genug: die Fufsblätter und der dreikantige Stengel sind wie beim Cyperus Papyrus ${ }^{1}$ ); der Kopf: ist an seinem Ansatz stets von $d r e i$ schmalen, langen Blättern von diesem Querschnitt ح umgeben, die mit einem kleinen, scharfen Knick sich vom Stengel loslösen. Sie sind stets so lang, dafs sie die rötlichbraun gefärbten, fuchsschwanzähnlichen Blütenstände trotz ihrer langen

1) Siehe: Ägyptische Pflanzensäule S. 25. 


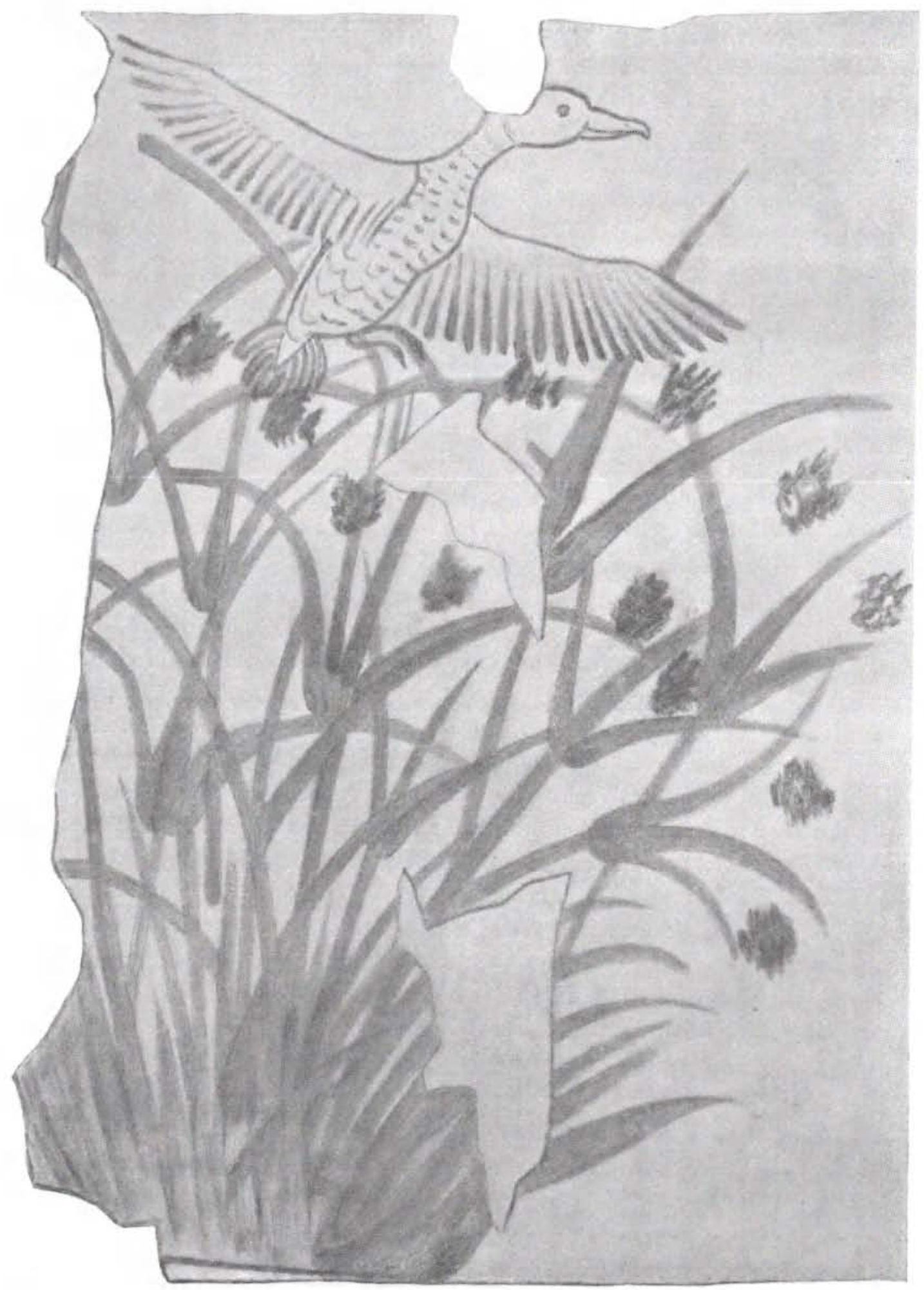

Abb. 3: Busch von Cyperus alopecurö̈des aus dèm bemalten Fufsboden von Hawata, jetzt im Berliner Musem. (Dynastie 18, Amenophis IV.) 
Doldenstrahlen überragen. Beim Cyperus alopecuroüdes ist es also umgekehrt wie beim Cyperus Papyrus, bei dem die Kopfblätter meist kürzer als die Doldenstrahlen sind und den Blütenstand daher mit ihren Spitzen nicht erreichen.

Darstellungen der Pflanze oder ihre

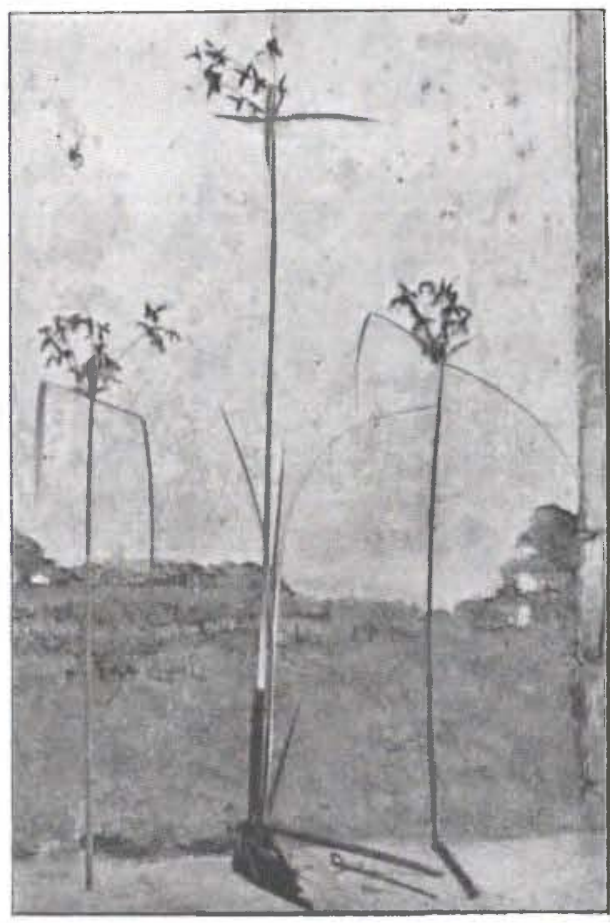

Abb. 2: Cyperus alopecuroïdes. ornamentale Verwendung kann ich augenblicklich aus den Zeiten des a. R. und m. R. nicht nachweisen, aus dem n. R. (Dynastie 18, Amenophis IV. und seine nächsten Nachfolger) dagegen haben wir sehr schöne realistische Abbildungen in den Fufsbodenmalereien von Et-Tell ${ }^{1}$ ) und Hawata (Abb. 3). Die Pflanze ist in diesen Abbildungen nicht zu verkennen und ist auch sofort bei Auffindung der Malereien identifiziert worden $\left.{ }^{2}\right)$. Die langen Fufsblätter streben spitz aus

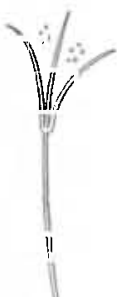

Abb. $4:$ Cyperus alopecuroüdes im naturalistischen Ornament. dem Wasser hervor und umgeben dicht die höheren Stengel, an deren Enden die drei Kopfblätter unter den Blütenständen scharf absetzend sich entwickeln und weit über die Blüten hinausragen. Diese Blüten sind (s. Abb. 4) durch rote Tupfen zwischen den Kopfblättern recht getreu wiedergegeben; hin und wieder werden auch einzelne der Doldenstrahlen mitgezeichnet. So frei wie in diesen Fursbodenmalereien findet sich nun unsere Pflanze in der Ornamentik ${ }^{3}$ ) natürlich nicht dar-

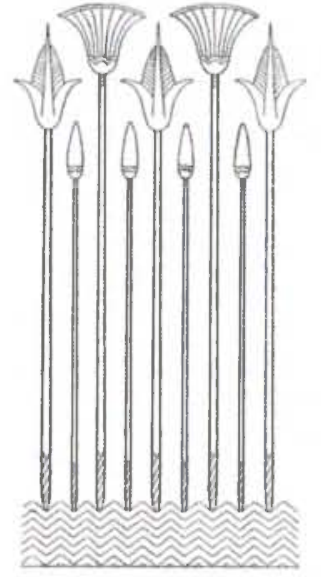

Abb. 6: Cyperus Papyrus und Cyperus alopecurö̈des als Sockelornament. (Nach Prisse.) gestellt. Der ornamentalen Verwendung und Stilisierung der Pflanze setzt sich dieselbe Schwierigkeit entgegen, die der Cyperus Papyrus darbot: der Blütenstand bietet nicht genug Masse. Beim Papyrus half sich der Ägypter dadurch, dafs er die ganze Dolde voll wiedergab, beim Alopecuroïdes ordnet er dagegen die grölseren, aber spärlicheren Blütenschwänzchen zu einer Masse

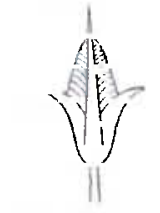

Abt: $:$ : Cyperus alopecuroüdes im stilisierten Ornament.
1) Pe'rie, Tell-el-Amarna Taf, 2 (viermal); Taf. 3, 2 Mitte und 3 Mitte; Taf. 4, 6 rechts.

2) Petrie, Tell-el-Amarna S. 13.

$\left.{ }^{3}\right)$ Auch in der mykenischen Kunst tritt die Pflanze ornamental, wenn auch in einiger Verwilderung, auf: \%. B. in der Decke von Orchomenos, auf dem einen Dolche aus Mykenä und vielleicht auf Topfscherben (Perrie, Tell-el - Amarna Taf. 27 Nr. 36 und 39, Taf. 28 Nr. 57). 
(s. Abb. 5). In dieser Form sehen wir die Pflanze sehr häufig im Ornament der Spätzeit, besonders an den Mauersockeln der Tempel ${ }^{1}$ ), wo sie reihenweise, mit noch geschlossenen Exemplaren oder mit anderen Wasserpflanzen abwechselnd, dargestellt ist (Abb. 6).

An der Säule ist die Pflanze ganz ebenso gebildet wie im Ornament. Beispiele dafür kann ich zwar bis in das Ende des n. R. hinein nicht nachweisen, dafür aber um so mehr aus der Spätzeit. Die hier abgebildete Säule aus Philä, der sich leicht eine ganze Reihe von Beispielen angliedern liefse ${ }^{2}$ ), zeigt in der oberen Pflanzenreihe ihres Kapitells Cyperus alopecuroïdes und Cyperus Papyrus in abwechselnder Folge. Die Fufsblätter sind beiden gemeinsam. Auch die Stengel des Alopecuroïdes sind wie die des Papyrus dreikantig $^{3}$ ). Die gleichfalls dreikantigen Kopfblätter überragen den Blütenstand weit, während sie beim Papyrus kurz zurückbleiben. Besonders scharf ist die Mittelrippe des mittelsten, gerade aufstrebenden Kopf'blattes betont. Die Blütenschwänzchen sind dicht aufeinander gepackt zwischen den Kopfblättern wiedergegeben. Bei den noch farbig erhaltenen Beispielen sind sie stets rot gemalt, während die Kopfblätter grün, hin und wieder auch blau erscheinen.

Im Anschlufs an vorstehenden Aufsatz möchte ich Gelegenheit nehmen, nochmals mit neuem Material auf die Thèse zurückzukommen, mit der ich seiner Zeit die Studie über die ägyptische Pflanzensäule schlofs. Ganz neuerdings hat noch W ILCKEN in dieser Zeitschrift ${ }^{4}$ ) klar und scharf zusammengefalst,

1) Mut-Tempel in Karnak, in Edfu, in Esneh, in Denderah, in Philä u. s. w.

2) Z. B. LD. I, 107a, 108, IV, VI und VII (farbig).

3) Nicht immer scharf wiedergegèben, wie z. B. beim Papyrus in der nebenstehenden Abbildung.

4) ÄZ. 1901, S. $66 \mathrm{ff}$.

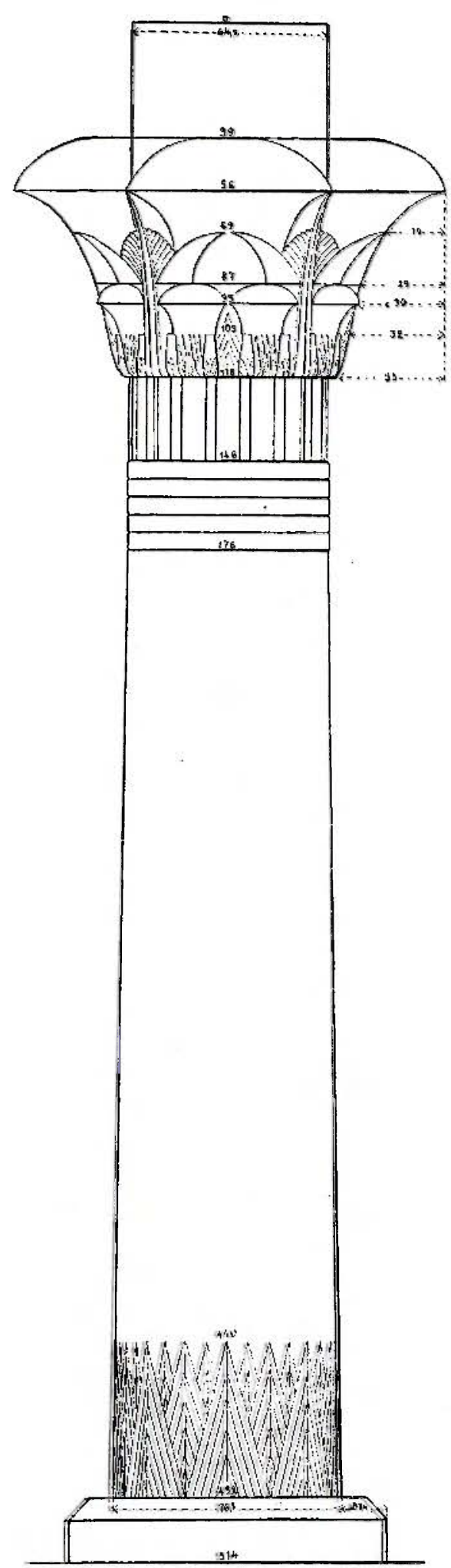

Abb. 7: Sãule aus dem Hofe des IsisTempels von Philä (Ptol.). Būndel von Cyperus Papyrus und Cyperus alopecuroüdes. 
was von anderen ${ }^{1}$ ) schon dagegen vorgebracht worden war, indem er die Frage auch von neuen Seiten beleuchtete.

Vor allen Dingen hat WILcken vermieden, auf das einzugehen, was so viele Unklarheit in die Deduktionen hineingetragen hat, nämlich auf den Ursprung der Säulen. Davon hatte ich absichtlich damals in meiner Arbeit nicht gesprochen, sondern nur davon, was sich die Ägypter unter ihren Pflanzensäulen vorstellten, welche Ideen sie ihnen zu Grunde legten. Ich glaube nämlich, dafs wir bisher bei unserem für die ältesten Zeiten notorisch mangelhaften Material noch nicht im stande sind, über den Ursprung der ägyptischen Pflanzensäulen etwas zu ermitteln. Nur das negative Resultat erhalten wir, dafs sie nicht aus der mit Pflanzen dekorierten Stütze entstanden sind.

Wäre das Vorbild der ägyptischen Pflanzensäule eine Blumendekoration um das Kapitell gewesen, so wäre eben daraus ähnliches entstanden wie bei der korinthischen Säule oder bei den gotischen Kapitellen. Man würde durch die Dekoration immer noch das Konstruktive des Kapitells hindurchsehen. Es wäre bei dieser Annahme auch kein Grund zu finden, der den Architekten gezwungen hätte, mit der Darstellung der Pflanzen so aus dem Mafsstabe zu fallen, wie es bei der ägyptischen Pflanzensäule geschehen ist, und es wäre vollends kein Grund vorhanden, die ganze Stütze, nicht nur das Kapitell, mit den Pflanzen zu verhüllen. Wie solche an ein Kapitell angebundenen Pflanzen aussehen, das können wir an der ägyptischen Pflanzensäule selbst beobachten, wenn wir die Zwischenstengel z. B. an geschlossenen Papyrusbündelsäulen betrachten, die in Proportionen, welche für das Steinmaterial ganz normale zu nennen sind, zwischen den Halsbändern stecken, ohne zu

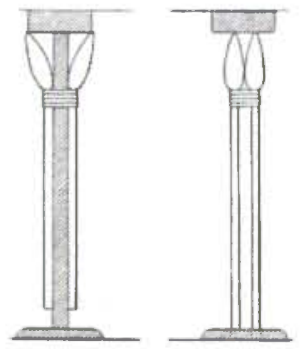

Abb. 8: a) Von angebundenen Blūten umhüllte "Pfeilersăule" .

b) Blütenbündel als Pflanzensăule. viel von dem Stamm, an den sie angebunden sind, zu verdecken. Aus solchen Elementen müfste das ganze Kapitell zusammengesetzt sein, wenn es aus einem dekorierten Konstruktionsteil entstanden wäre.

Auch würde diese Entstehungsart die starke Schwellung des Säulenfưfses bei den Papyrus-Bündelsäulen nicht erklären, die nur natürlich erscheint, wenn man annimmt, die Bündelsäule stelle eben einen Büschel eng im Boden zusammenstehender Pflanzen dar, deren Stengel auseinander streben wollen, aber oben gewaltsam durch ein Band zusammengefafst sind.

Ferner würde ein Säulenkopf, der mit Blumen geschmückt ist, nie ein solches Profil zeigen können wie die Kapitelle der geschlossenen Nymphäen- und Papyrussäulen. Es würden sich stets nach oben divergierende Profillinien (Abb. 8a) ergeben habeñ, nie konver-

1) BeI.GRr in Philol. Wochenschr. 1899, S. $467 \mathrm{ff}$., Naville in Sphinx II, S. $224 \mathrm{ff}$. Die einzigen mir bekannt gewordenen Kritiken von fachmännischer Seite (Hofmans bez. Dörpfecd in Deutsche Bauzeitung 1899, S. 28) schliefsen sich den von philologischer Seite vorgebrachten Bedenken nicht an (vergl. hierzu Pient in Sphinx VI, S. 48). 
gierende (Abb. 8b), die in eine Spitze endigen, auf der der überstehende Abakus balanciert. Dieser von der Pflanzensäule völlig losgelöste Abakus hat auch WinckeN bei der Ausführung seiner Theorie Schwierigkeiten bereitet, und daher nimmt er an, dafs sowohl Abakus wie Basis nicht zu der ornamentalen Pflanzensäule gehören, dafs sie vielmehr besondere Teile der in dem Blumenschmuck steckenden "Pfeilersäule " wären. DaIs beide Teile konstruktiven Notwendigkeiten ihre Entstehung verdanken, halte auch ich für sicher. Der Abakus ist die Auflagerplatte für den Architrav, seine Form ist durch den darüber hinlaufenden Balken gegeben, und mit dieser Form hat der ägyptische Architekt bei der Ausbildung der Pflanzensäule ornamental nichts anzufangen gewulst. Der Abakus ist ihm eben nur die rein konstruktive Verbindung zwischen dem mit dem Kapitell abgeschlossenen Pflanzenbüschel und der darüber schwebenden Himmelsdecke. Die Basis wiederum ist die Druckplatte, die den Druck der Säule auf den Erdboden zu übertragen und zu verteilen hat; diese hat der Architekt seiner Gesamtidee noch anzupassen gewufst, indem er daraus den Erdhügel bildete, den er in der Natur um den Fuls der von ihm dargestellten Pflanzen so oft beobachtet hatte, wenn das Überschwemmungswasser abgelaufen war. Bei Palmen namentlich wird er häufig den Erdhügel bei den im Trocknen stehenden Exemplaren gesehen haben ${ }^{1}$ ), bei den Wasserpflanzen ist diese Erdansammlung allerdings meist vom Wasser bedeckt. Das hinderte den Architekten aber nicht, sie doch zur Darstellung zu bringen. Auf diesen Fall werden wir noch unten zurückkommen.

Die Natur der Nymphäen, meint Wilcken, spräche schon dagegen, dal's man annehmen könne, dieselben strebten ohne festen Anhalt an einen inneren, nicht pflanzlichen Kern allein gen Himmel. Ein Nymphäenstengel liebe posthornartige Windungen, - aber nur wenn die Pflanze ausgerupft ist. So lange sie im Boden steht, strebt der Stengel kerzengerade aus dem Wasser heraus und trägt stolz seinen Blütenkopf bis zu dem Moment, wo die Pflanze abblüht und welkt. Hätte man ausgerupfte Pflanzen um einen Pfahl gebunden ${ }^{2}$ ), so wären wahrscheinlich die posthornartigen Windungen der Stengel auch in der Ornamentik geblieben. Es wäre sogar zu vermuten, dafs dann die Blüten keine aufstrebende, sondern nach abwärts sich bewegende Richtung erhalten hätten, etwa wie die Nymphäen in Friesen ${ }^{3}$ ) oder wie der Papyrus in dem häufigen Ornament aus zwei zusammengebundenen $\operatorname{Dolden}^{4}$ ).

1) Siehe Pflanzensäule S. 44, Abb. 71.

2) Auf die ÅZ. 1901, S. 86 angeführten Beispiele für mehrere zusammenhaltende Bänder, welche die Posthörnchen gerade halten sollen, ist es besser, nicht einzugehen. Perrot-Chipie\% S. 489 = LD. II, 61 giebt wohl nur Farbenteilung, wie etwa bei den Beispielen aus Benihassan (Pflanzensäule S. 7 und 8); Perrot -Chipiez S. $503=$ Descr. III, 8 ist heute in Luqsor nicht mehr nachweisbar und wird es auch 1800 nicht gewesen sein; und endlich Perrot-Chiprez S. 505 zeigt nur Inschriftstreifen, die als Abschlüsse zu anorganisch auf die Säule gesetzten Bildern gehören. Etwa sonst vorkommende vervielfachte Bindungen sprechen übrigens keineswegs gegen die oben verteidigte Auffassung der Bündelsäule.

8) Siehe Pflanzensäule S. 13, Abb. 22.

4) Siehe Pflanzensäule S. 28, Abb. 48 und 49 . 
In der Frage, ob das Bündel oder die Einzelpflanze das Primäre wäre, entscheidet sich WILckeN für das erstere, und ich stimme ihm teilweise darin $\left.z^{1}\right)$. Wir haben zwei in Beispielen erhaltene Arten von Einzelpflanzensäulen: die offene Papyrussäule und die Palmensäule. Für die Papyrussäule möchte ich meine 1897 ausgesprochene Ansicht beibehalten, dals sie nämlich die Kopfbänder von Bündelsäulen der gleichen Gattung entlehnt habe, von deren Vorkommen uns Beispiele der Spätzeit unterrichten, dafs sie also selbst als eine sekundäre Erscheinung zu betrachten sei. Die Palmensäule dagegen kann ich nicht als etwas Sekundäres ansehen, da eine Palmenbündelsäule, die als das Primäre anzunehmen wäre, überhaupt nie existiert hat. Ich möchte heute auch für die Kopfbänder der Palmensäule eine andere Deutung vorschlagen als damals ${ }^{2}$ ). An ein Anbinden von Palmblättern an eine "Pfeilersäule “ ist nicht zu denken, da die späten Beispiele zeigen, wie die Blätter und Früchte natürlich aus dem borkigen Stamm herauswachsen. Auffällig ist, dals nur bei den Palmensäulen, und zwar hier schon bei den ältesten Beispielen aus dem Totentempel des Königs Onnos (Dynastie 6), unter den Kopfoändern eine Art herabhängender Schleife angebracht ist. Darin glaube ich jetzt die Stricke sehen zu müssen, mit deren Hilfe die Palme zur Zeit der Dattelreife — in der Überschwemmungszeit - bestiegen wird. Der Bauer, der seine Palme aberntet, sitzt auch heute noch, die Füfse gegen den Stamm stützend, in einer ähnlichen Schlinge unter der Krone.

Die Frwähnung der Überschwemmungszeit bringt uns nun auf eines der besten Argumente des Wilckenschen Aufsatzes: der Erdhügel, aus dem die Wasserpflanzen hervorwachsen, mülste sich unter Wasser befinden, und "man dürfte erwarten, dafs z. B. in der berühmten Fufsbodenmalerei zu Tell-el-Amarna das Wasser des 'Teiches über die Standplätze der Säulen hin ausgedehnt wäre “. Sehen wir uns darauf einmal den Fufsboden von Et-Tell an ${ }^{3}$ ). Wie er sich jetzt zeigt, ist er die Erneuerung eines anderen, vielleicht wenige Jahre vorher verlegten, bald abgetretenen Bodens ${ }^{4}$ ), der eine ganz andere Anordnung und Einteilung hatte wie der jetzt sichtbare. Bei diesem ursprünglichen Fulsboden stehen, wie man bei der zweiten Säule von Westen in der südlichen Reihe ${ }^{5}$ ) deutlich sehen kann, die Säulen im Wasser, während sie bei dem zweiten, übergemalten Entwurf in der Reihe der am Rande der Teiche wachsenden Wasserpflanzen stehen. Leider können wir aber nicht mit Bestimmtheit angeben, welchen Pflanzen die Säulen dieses Saales nachgebildet waren. Nach den von

1) Siehe Pflanzensäule S. 40 und 46.

2) Siehe Pflanzensäule S. 46.

3) Perrie, Tell-el-Amarna Taf. 2.

4) Der untere Boden besteht aus einer 2,5 cm dicken, grauen Möıtelschicht, auf der der erste, bemalte, $0,5 \mathrm{~cm}$ starke Gipsestrich liegt. Bei der Erneuerung wurde nur ein zweiter, wiederum $0,5 \mathrm{~cm}$ starker Gipsestrich aufgelegt, der dann frisch bemalt wurde. (Vergl. PETRIE, Tell-el-Amarna S. 13.)

5) Perrie, Tell-el-Amarna Taf. 2 und Taf. 4 Nr.5. 
Petrie gefundenen Fragmenten ${ }^{1}$ ) können es Wasserpflanzen, nach den in den Gräbern erlıaltenen Abbildungen ${ }^{2}$ ) des Saales können es Palmen gewesen sein. Das létztere würde für WILckens Argument auch kein Hindernis sein, da die Palmen während der Überschwemmungszeit auch im Wasser zu stehen pflegen. Jedenfalls sehen wir aber, dafs der Architekt, der den Fufsboden erneuerte, sich kein Gewissen daraus machte, Pflanzensäulen, die früher in dem gemalten Wasser standen, nun an den Rand des Tanks zwischen die gemalten Wasserpflanzen zu stellen. Es mufs also beides nicht gegen die Auffassung der Pflanzensäule verstolsen haben.

Ich bin sogar der Ansicht, dafs, wie Wrucken ganz richtig folgert, die Angabe des Wassers, aus dem die Pflanzensäule herauswächst, in der Architektur sich zeigen muls, und will dies im folgenden durch einige Beispiele belegen, die ich im Laufe der letzten Jahre dafür gesammelt habe. Um nicht den Schein zu erwecken, als sei diese Materialsammlung ad hoc für die Widerlegung des Wirckenschen Aufsatzes gemacht worden, gebe ich die Thatsachen in der an und für sich nebensächlichen Reihenfolge, in der sie sich mir ihrer Zeit selbst darboten.

Im Februar 1898 fiel mir an den Resten von Papyrussäulen — vermutlich mit offenen Dolden - in Kom-el-hetan (Dynastie 18, Amenophis III.) auf, dafs dieselben in geringem Abstand über der Basis Spuren einer Reihe von Metallnägeln zeigten, die von einem dünnen Metallbeschlag herrühren mufsten, der in etwa $10 \mathrm{~cm}$ Breite den Papyrusstamm an seinem unteren Ende dicht über der Basis umgeben hatte. Weitere Beobachtungen zeigten, dafs dieser Streifen an allen Papyrussäulen wieder auftritt, nur nicht in Metall ausgeführt, sondern in Stein, ohne jedes Relief. Es ist bei den meisten Säulen eigentlich weiter nichts als ein unten um die Säule direkt über der Basis herumlaufender glattgelassener Streifen, der oben durch eine eingekratzte Linie begrenzt wird und über dem erst die Fulsblätter des Papyrus ansetzen. An einigen hier folgenden, historisch geordneten Beispielen mag man sich von dem Vorhandensein dieses bis jetzt merkwürdigerweise unbeachtet gebliebenen Streifens ${ }^{3}$ ) überzeugen:

Die älteste Papyrussäule, die zur Zeit bekannt ist, die sechsfache Bündelsäule vom Totentempel des Ne-woser-rec (Dynastie 5) bei Abusir ${ }^{4}$ ), hat in $3,5 \mathrm{~cm}$ Breite den glatten Streifen über der Basis.

An der nächstältesten, der achtfachen Bündelsäule vom Totentempel Amenemhêts III. (Dynastie 12) bei Howara ${ }^{5}$ ), ist die Linie heute nicht mehr nachweisbar, da die unteren Teile der Säule abgebrochen sind.

1) Siehe Perrie, Tell-el-Amarna S. $9 \mathrm{ff} . \quad$ 2) Siehe Pflanzensäule S. 54, Anm. 3.

${ }^{3}$ ) Papyrusstengel als Spiegelgriffe (Berl. Mus. Nr. 2774, 2818; de Morgan, Dalichour Taf. 20, Nr. 24) oder Amulette (Berl. Mus. Nr. 6462, 6464, 6470) u. s. w. haben den Streifen nicht.

4) Mitteilungen der Deutschen Orient-Gesellschaft Nr.14, Blatt 3.

5) Pflanzensäule S.32 nach LD. I, 47. Die ERBкamsche Aufnahme, der auch die Angabe der Stelle für die Inschrift fehlt, die diese Säule wie die von Abusir trug, hat das untere Ende der Säule wohl falsch ergänzt. 
An der achtfachen Bündelsäule Thutmosis' III. (Dynastie 18) vor der kleinen Kapelle südlich vom Pylon von Luqsor findet sich der Streifen gleichfalls ${ }^{1}$ ).

Die achtfachen Bündelsäulen aus der Zeit Setis I. (Dynastie 19) in Soleb ${ }^{2}$ ) haben einen besonders hohen Streifen.

An den abgedrehten Bündelsäulen aus der Zeit Setis I. und Ramses' II. (Dynastie 19) in $\mathrm{Abydos}^{3}$ ) und in Karnak ${ }^{4}$ ) findet sich der Streifen gleichfalls,
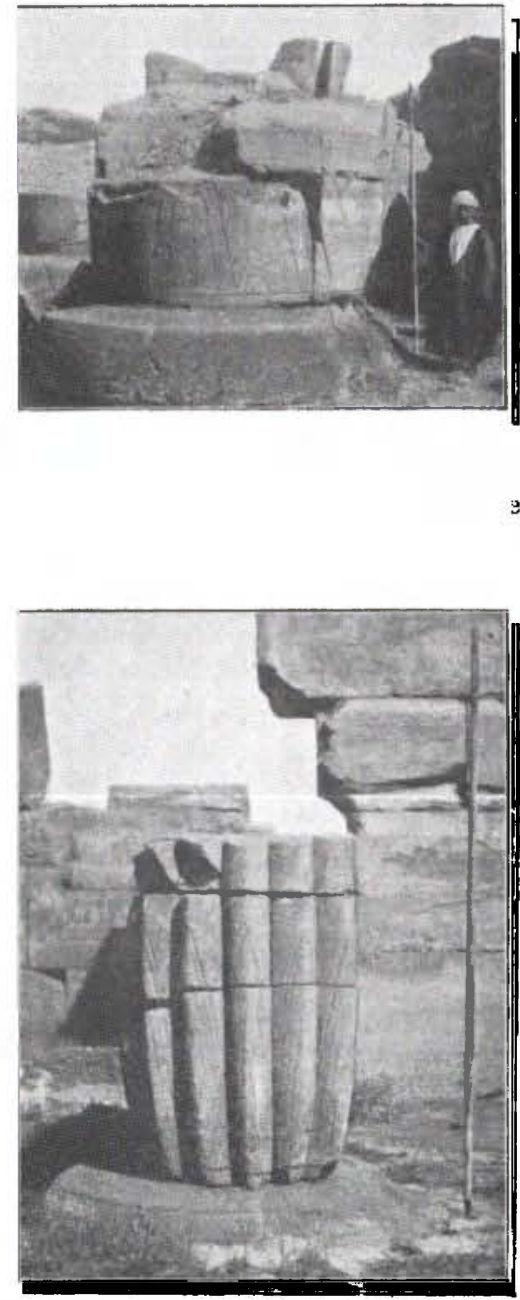

Abb. 10:

Säulenfứs einer Papyrusbündelsäule aus Karnak ${ }^{7}$ ) (Dynastie 18). ebenso an den Säulen aus der Zeit Ramses' II. (Dynastie 19) im Ramesseum ${ }^{5}$ ) und an denen aus der Zeit Ramses' III. (Dynastie 20) in Medinet $\mathrm{Habu}^{6}$ ).

Kurz, es giebt meines Wissens keine Papyrussäule, welche jenen Streifen nicht aufweisen könnte. In der Spätzeit kommen wohl einige Beispiele vor, die ihn nicht haben. Aber die Spätzeit setzt sogar Fufsblätter um Palmensäulen; also ist auf das Fehlen des Streifens in dieser Zeit nichts zu geben.

Dals der Streifen nun nicht etwa als Band zu erklären ist, ergiebt sich erstens daraus, dals er nicht profiliert (Abb.9) ist, wie es die Kopfbänder unter dem Kapitell stets sind, und dafs er ferner nicht die Gesamtheit der Stengel zusammenfalst, sondern den Stengelprofilen folgt, wie am deutlichsten an den zwölffachen Bündelsäulen beim sechsten Pylon von Karnak (Abb. 10) zu sehen ist.

Da es also kein Band sein kann, so nahm ich zuerst, wenn auch nur zögernd, an, dafs dies die Angabe des Wassers sein sollte, aus dem die Pflanzen hervorwachsen, und das die Basis, d. h. den Erdhügel, der die Wurzeln deckt, überspült. Diese Annahme wurde mir zur Gewilsheit, als ich im Juni 1900 bemerkte, dals an den Papyrussäulen des ersten Saales im grofsen Tempel von Abydos der betreffende Streifen deutliche Reste blauer Bemalung zeigte, die sich, wenn auch nicht mit gleicher Sicherheit, auch auf der Basis verfolgen lielsen. Infolge dieser Beobachtung wurde es nun notwendig, die Farbenreste an den Säulenbasen genauer anzusehen. Zuerst versuchte ich dies an den Ab-

1) Photographie Beato.

2) LD. I, 117, Säule X. In der Zeichnung des Såulenfufses sind einige Darstellungsfehler zu verbessern.

$\left.{ }^{3}\right)$ Photographie Beato. $\quad{ }^{4)}$ Pflanzensäule S. 35 nach LI). I, 80.

5) LD. I, 90.

B) Photographie Beato.

¡) Die "Wasserlinie" ist durch Retouche etwas hervorgehoben. 
bildungen, mit denen aber, wie sich bald zeigte, nichts anzufangen war. Wenn schon bei gewöhnlichen, unkolorierten Aufnahmen unsere Veröffentlichungen fast nie einen Unterschied zwischen Gesehenem und Ergänztem machen, so ist dies bei farbigen Darstellungen noch viel mehr der Fall. Die Farbenspuren an Säulen sind eben so geringe, dafs nur in den seltensten Fällen so farbige, volle Bilder herauskommen würden, wie sie unsere Veröffentlichungen geben. Ganz besonders sind aber die in den Zeichnungen angegebenen Farben an den Säulenbasen verdächtig, da in natura fast nie mehr Farbenreste an den Basen zu sehen sind. Wenn man also sicher gehen wollte, so mufste man die Veröffentlichungen ganz Seite lassen und an den Originalen suchen. Ein gewisses Resultat mufste z. B. bei an den seit dem Ende des n. R. ummauerten Säulenbasen der Vorhalle des Amonstempels Amenophis' III. (Dynastie 18) zu Luqsor zu erwarten sein. Als Stücke des Mörtels, der die betreffende Stelle verschmiert hatte, vorsichtig entfernt wurden, trat hier wirklich an dem glatten Streifen zwischen der Basis und den Fufsblättern das leuchtendste Blau zu Tage. Beim weiteren Suchen wurden noch an den Säulen des westlichen Saales im Ramesseum (Dynastie 19) sehr dunkle blaue Farbspuren ${ }^{1}$ ) an denselben Stellen und mit geringerer Sicherheit auch auf der Basis nachgewiesen.

Andere Farbenspuren an den Basen oder an den glatten Streifen dicht über denselben habe ich bei meinem Suchen bisher nicht gefunden. An den paar Stellen, an denen Farbe nachgewiesen wurde, ist sie also blau. Ich will aber trotzdem annehmen, dals das Gelbbraun, das die Publikationen den Basen geben, das ich aber in natura noch nirgends wiedergefunden habe, dennoch vorkommt. Das würde die Erklärung des blauen Streifens über der Basis als Angabe des Wassers dennoch nicht hindern. Es wäre dann eben eine solche Bemalung, wie wir sie z. B. im a. R. ${ }^{2}$ ) bei den Darstellungen von Menschen und Tieren finden, welche durch Wasser gehen. Die blaue Farbe des.Wassers wäre dann nur an der Oberfläche durch einen breiten Streifen angegeben; darunter erscheint wieder die eigentliche Farbe des durch das Wasser durchschimmernden Gegenstandes, an den Säulen also das Gelbbraun des Erdhügels, in den obengenannten Bildern das Rotbraun der menschlichen Hautfarbe.

Aufser der blauen Färbung auch noch Wasserlinien auf oder über der Basis zu verlangen, wie WrLcKEN es thut, ist für die neue Auffassung meines Erachtens nicht unumgänglich notwendig, jedoch könnte sich dies auch noch einst auf irgend einem Beispiele zeigen. Vielleicht ist ein Zickzackornament, das sich oben auf der Basis einer Säule bei Pylon 6 in Karnak findet (Dynastie 18), aus solchen Wasserlinien entstanden.

Das Vorstehende wird hoffentlich zur Genüge gezeigt haben, dafs die alten Architekten sich die Pflanzen, welche ihre Säulen bildeten, wirklich als aus dem Wasser hervorwachsend dachten, dals sie also einen Saal mit Pflanzen-

1) Die Publikation LD. I, 90 giebt dieses dunkle Blau als Grün wieder.

2) Z. B. im Grabe des Ty, Kammer G (nach BAEdeker), Nordwand neben der Thür. 
säulen nicht "á l'image du monde", allgemein gefafst. darstellten, sondern als ein Abbild der Welt in einer ganz bestimmten, für Ägypten besonders wichtigen Jahreszeit, während der Überschwemmung. Ich will solchen Saal daher "Überschwemmungssaal " nennen. In ihm ist ein Stückchen Erde alogebildet, etwa wie es die unten beigegebene Abbildung einer Landschaft aus der Gregend von Biahmu im Faijum (Abb. 11) wiedergiebt.

Am Boden des Raumes sind fischreiche Lachen der Nilüberschwemmung dargestellt, die von allerhand Wasserpflanzen umgeben sind. An den Wandsockeln ziehen sich Reihen solcher Wasserpflanzen hin, unter denen die Angabe der Wasserlinien, oft auch kleiner Bassins, nie vergessen ist. Aber auch aus der Mitte der Teiche und an ihrem Rande spriefsen Büschel von Cyperus-

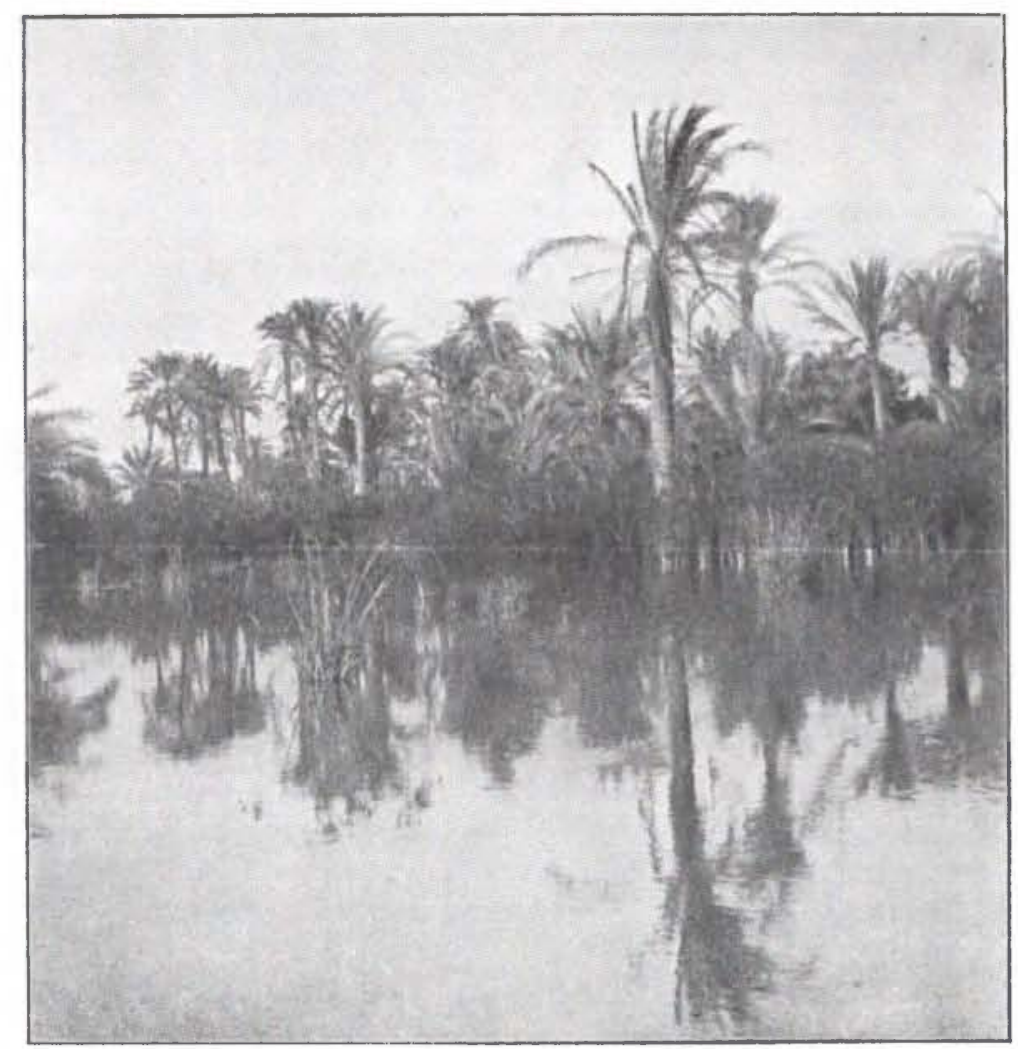

Abb. 11: Überschwemmungslandschaft aus dem Faijum.

und Nymphäenarten. Oft sind das vielleicht auch wirkliche, lebende Pflanzen gewesen, wie ich aus dem Vorkommen von vertieften Bassins im Fufsboden des Palastes von Hawata ${ }^{1}$ ) schliefsen möchte, immer aber sind es die aus konstruktiver Notwendigkeit in Holz oder Stein umgesetzten Pflanzensäulen, diese mit thunlichster Naturwahrheit aufgebauten Büschel von Wasserpflanzen, unter die sich hin und wieder auch Einzelpflanzen, wie Palmen oder ein einzelner

$\left.{ }^{1}\right)$ Leider fehlt bisher jede Veröffentlichung dieses wichtigen, bereits 1897 vom Service des antiquitès aufgehobenen Fufsbodens. 
Papyrus, mischen. Das Auseinanderstreben der Büschel kann der Architekt in seinem Material nicht nachahmen, und so greift er denn zu einem Mittel, das er in der Wirklichkeit wohl oft vom Gärtner angewendet gesehen hat: er falst die einzelnen Stämme des Büschels durch Bänder unter den Köpfen zusammen und giebt dem Ganzen so eine seinen Baumaterialien mehr angepafste Form. Tierbei beobachtet er sogar solche Feinheiten, dafs er sclbst die Schwcllung richtig wiedergicbt, dic im unteren Ende eines am Kopf zusammengefafsten, sonst frei wachsenden Papyrusbüschels auftritt. Oft mögen auch wohl Jäger derartig die Büschel zusammengebunden haben, um daran ihre Jagdbeute bis zum Abholen aufzuhängen. Solchen Beohachtungen haben dann vielleicht die merkwürdigen Dekorationen mit gefangenen Vögeln ${ }^{1}$ ), die von den Halsbändern der Säulen herabhängen, ihren Ursprung zu verdanken. Ob das Einsetzen der Zwischenstengel etwa auch auf eine wirkliche Beobachtung zurückzuführen ist,

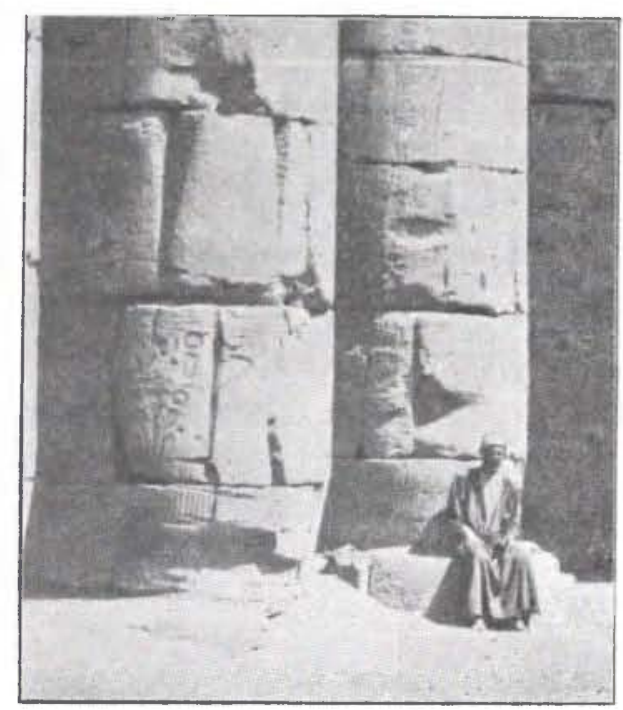

Abb. 12: Säulenfufs aus dem Hofe des ChonsTempels bei Karnak (I)ynastie 19).

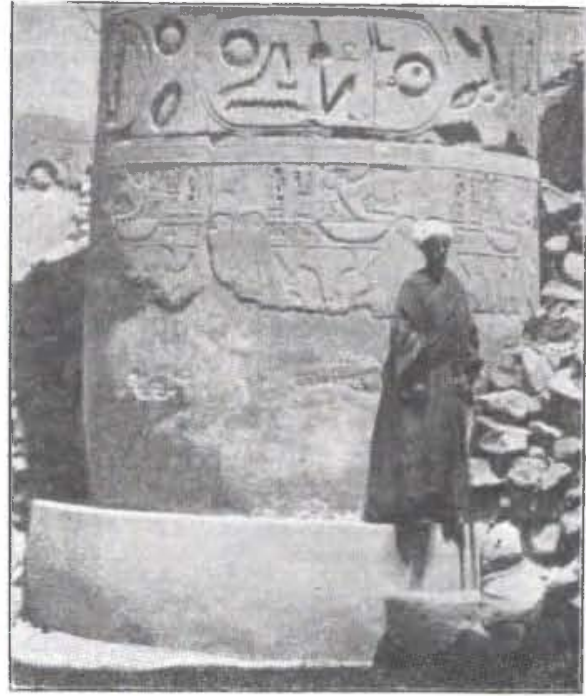

Abb. 13: Säulenfứs aus der grơsen Säulenhalle im Amons-Tempel bei Karnak (Dynastie 19).

dals etwa die Bänder besser festsafsen, wenn solche Bündelchen dahintergesteckt wurden, mag dahingestellt bleiben ${ }^{2}$ ).

Dafs er mit dem Halsbande nur die längsten Stengel des Büschels zusammenhalten kann, ist dem alten Architekten übrigens auch zum Bewufstsein gekommen. Er läfst daher kleinere, freie Stengel neben den grofsen, zusammengefafsten aufspriefsen und bringt diese, natürlich nur so grofs, dafs sie ihm die sonstige Dekoration seines Säulenschaftes nicht stören, am Fufse der Säule zwischen den Fufsblättern der grofsen Stengel an, nicht körperlich, aber in

$\left.{ }^{1}\right)$ LD. III 106c; Peirie, Tell-el-Amarna Taf. 7, Taf. 11, 6.

2) Das älteste Beispiel der Papyrusbündelsäule zeigt keine Zwischenstengel. 
feinem Relief oder in farbiger Zeichnung ${ }^{1}$ ). Auf diesen frei spriefsenden Stengeln hat er auch Platz, hier und da ein Vogelnest anzubringen, um die Naturnachahmung möglichst weit zu treiben. Beispiele hierfür sind häufig; zwei mögen für viele genügen: Säulen aus dem grofsen Saal in Karnak und die aus dem Hofe des Chons-Tempels ebendaselbst (Abb.12 und 13, beide Dynastie 19).

Für diese "Überschwemmungshalle", die wir soeben beschrieben haben, glaube ich nun auch den alten Namen in der Litteratur gefunden zu haben. Es stehen mir zur Zeit drei Stellen dafür zur Verfügung:

1. Pap. Westcar VIII, Z.9, 19 und 20: 6.

2. Pap. Sinuhe Z. 251: 813 吗

3. Ostrakon (Dynastie 20) in Kairo:

Aus den ersten beiden Stellen geht hervor, dafs dieses Wort W.3hj einen Raum des Palastes bezeichnet, in dem Audienzen stattfanden; aus dem Zusammenhange der zweiten Stelle glaube ich schliefsen zu dürfen, dafs dieser Raum sich vor dem König gewöhnlich thront ${ }^{3}$ ). Es wird also ganz gut passen, wenn wir den grofsen Saal des Palastes bei Et-'Tell, eben den, dessen gemalter Fufsboden uns auf die richtige Deutung der im Wasser stehenden Pflanzensäule brachte, als eine 8 蚂 Übersetzung möchte ich nämlich dem Worte geben im Hinblick auf die Bedeutung der Wurzel fou wih "überschwemmen ", über welche uns SETHE vor kurzem ${ }^{4}$ ) aufgeklärt hat.

$\mathrm{Ob}$ auch Worte wie

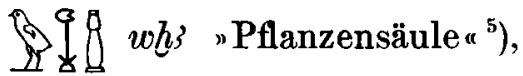

$$
\begin{aligned}
& \text { T) } 48 \text { whsj "Pflanzensäule “ }{ }^{6} \text { ), } \\
& \text { fi }
\end{aligned}
$$

1) Eine bekannte Analogie zu dieser Art der Darstellung zeigen die Fayence-Nilpferde aus den Gräbern des m. R., denen auch die Wasserpflanzen, zwischen denen sie herumpatschen, auf den Leib gemalt sind.

2) Die Schreibung 8$\}$

3) Nach früheren Ausführungen könnte es also eine andere Bezeichnung für "den breiten Saal « sein, der vor dem ntiefen Saal « des, Palastes lag. Ich würde also jetzt die Teile des Palastes wie folgt bezeichnen (vergl. ÄZ. 96, S. 108):

1. Vorhalle, ägyptischer Name mir unbekannt.

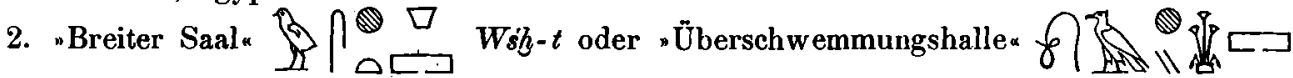
Wihj; der erste Name von der Form, der zweite von der Dekoration des Raumes hergenommen.

3. Tiefer Saal " W

4) ÄZ. 1900, S. 106.

6) Mar., Cat. d'Ab. 545.

5) Brugsch, Wb. Suppl. S. 327.

${ }^{7}$ Brugsch, Wb. S. 271. 
Tafel II.

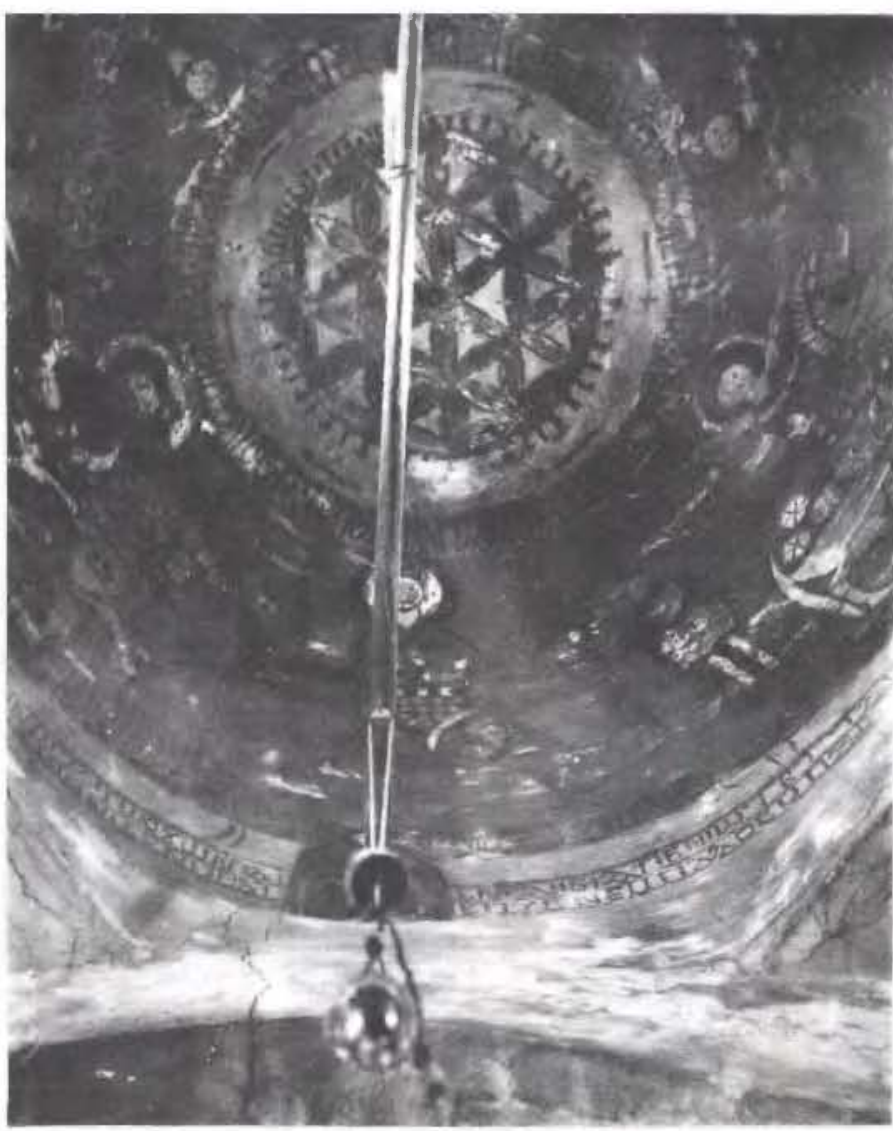

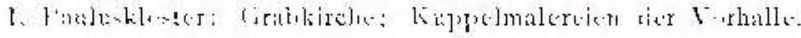

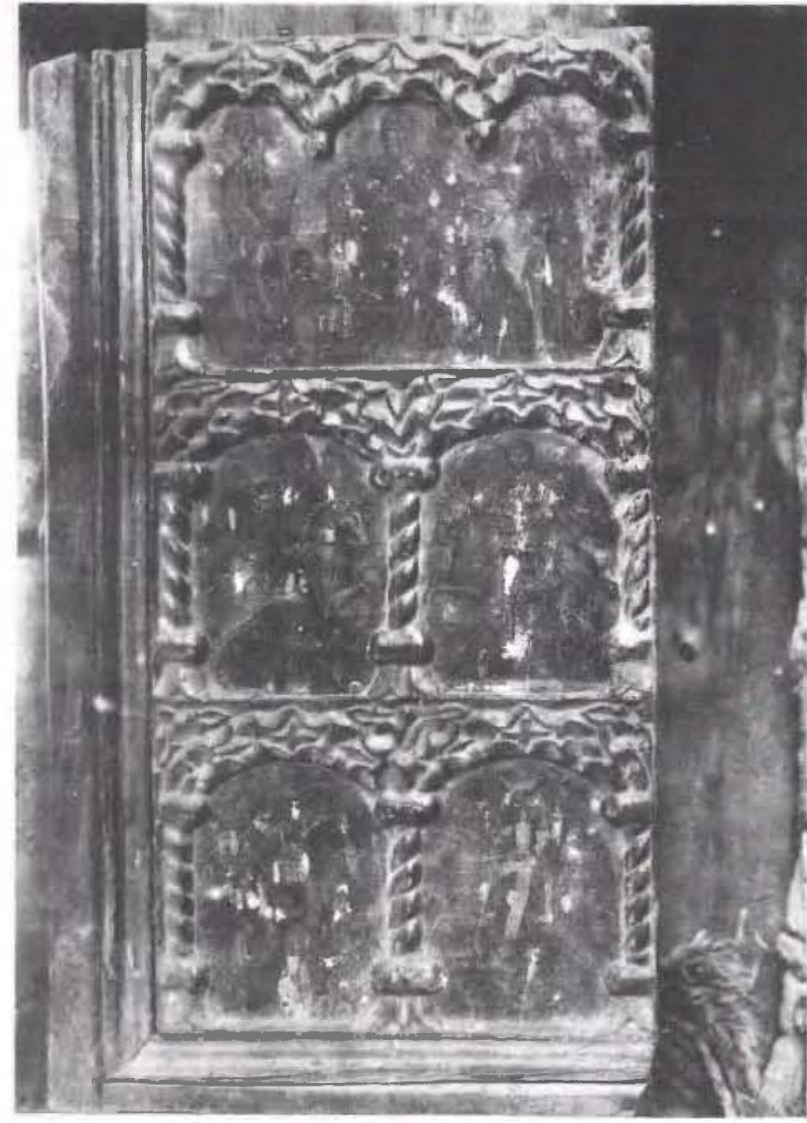

3. Antoniuskloster: Tafelbild des febna Dengel.

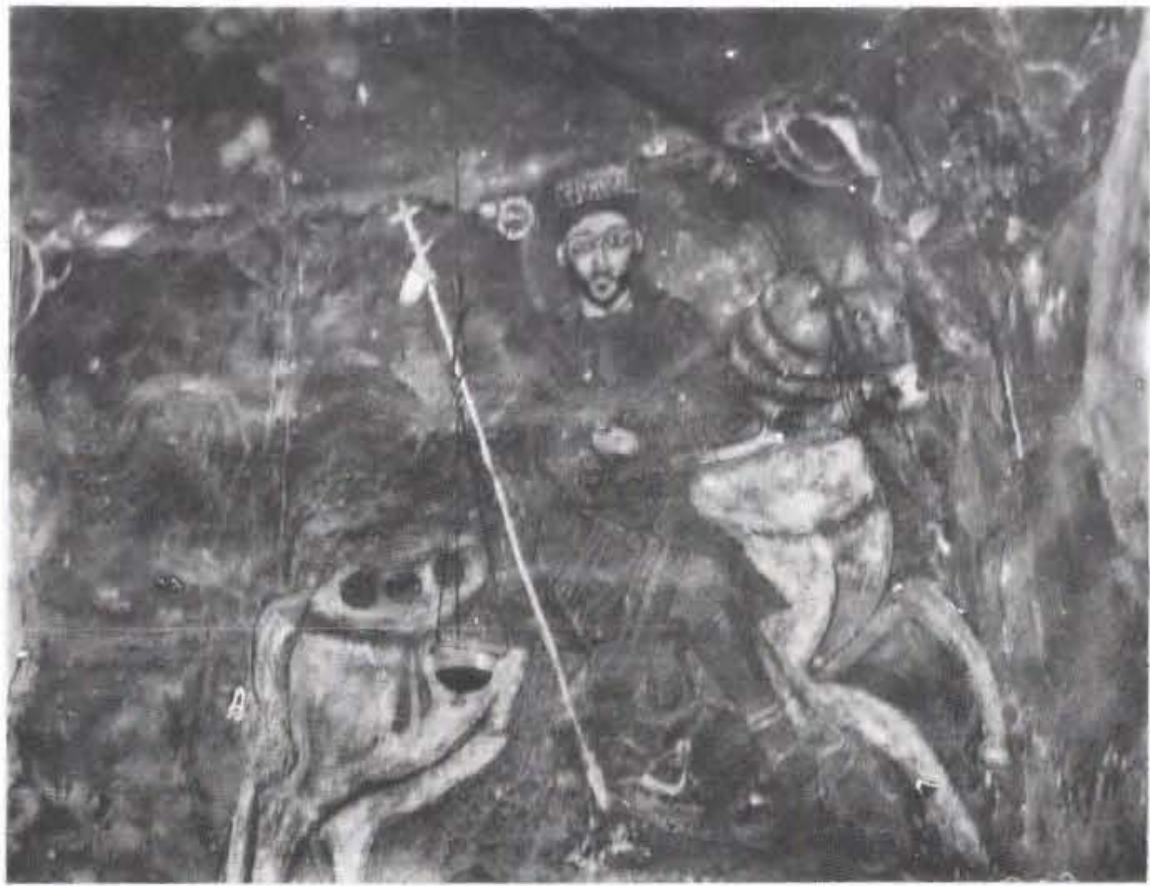

2. Antuniukluster: Reiter an den Wandmalercien der Kirche dew hl. Antonius.

Stryyowski, 1)er Koptische Reiterheilige und der hl. Georg. 
Brought to you by | University of Texas at El Paso 
auf denselben Stamm zurückgehen, mögen andere entscheiden, denen solche Fragen geläufiger sind als $\left.\operatorname{mir}^{1}\right)$. Dafs diese Worte auch mit dem Stein $\square$ und dem Holz $\hookrightarrow$ determiniert vorkommen ${ }^{2}$ ), spricht nicht gegen ihre Ableitung vom Stamme 8 wh "Überschwemmen", sondern ist nur als sekundäre Erscheinung so zu erklären, dafs in diesen Fällen die Pflanzensäule bez. der Säulensaal durch die Determinative ihres Materials näher bezeichnet werden.

Wie der ägyptische Architekt dazu gekommen ist, Säle in Palästen und Tempeln so zu dekorieren, dafs sie das Bild eines Überschwemmungsgebietes geben, das entzieht sich unserem Wissen vorläufig. Mögen dabei religiöse Vorstellungen insofern eine Rolle gespielt haben, dafs ein solcher Saal die Deltasümpfe wiedergeben sollte, in denen der junge Horus seine Kindheit verbrachte, und dafs man es nun für angebracht hielt, solche Dekorationen im Palaste des "lebenden Horus“, des Königs, zu wiederholen, oder mag die ganze Dekoration nur auf die übermächtig nachwirkende Schöpfung der Phantasie eines alten, besonders hervorragenden Architekten zurückzuführen sein, das werden wir wohl nie entscheiden können. Das für uns'Wesentliche daran ist nur, einzusehen, dafs die ägyptische Pflanzensäule nur einer rein dekorativen Idee ihren Ursprung verdankt, und dals die Konsţruktion, das Tragen, in ihr überhaupt nicht zum Ausdruck kommt. So bleibt also der Satz bestehen:

"Der Ägypter dachte sich seine Pflanzensäulen als freie Endigungen und ornamentierte sie wie solche."

\section{Der koptische Reiterheilige und der hl. Georg.}

\section{Von Josef Strzygowski.}

\section{Hierzu Tafel II.}

In dem eben erschienenen Hefte $\mathrm{V}$ des Bulletin de la société archéologique d'Alexandrie behandele ich unter dem Titel "Hellenistische und koptische Kunst in Alexandria " an der Hand einiger in Ägypten gefundener Beinschnitzereien und der Elfenbeinreliefs der Domkanzel zu Aachen u. a. auch jenen Heiligentypus,

1) SE'THE, Verbum I $\$ 87$.

2) LD. II, 125 Z. 192 und sonst. 